\title{
Obesity and telomere status in the prognosis of patients with colorectal cancer submitted to curative intention surgical treatment
}

\author{
SERGIO GARCÍA-MARTÍNEZ ${ }^{1}$, DANIEL GONZÁLEZ-GAMO ${ }^{1}$, \\ TAMARA FERNÁNDEZ-MARCELO ${ }^{1,2}$, SOFÍA TESOLATO ${ }^{1}$, SOFÍA DE LA SERNA ${ }^{3,4}$, \\ INMACULADA DOMÍNGUEZ-SERRANO ${ }^{3,4}$, OSCAR CANO-VALDERRAMA ${ }^{3,4}$, ANA BARABASH ${ }^{2,4,5}$, \\ CARMEN DE JUAN ${ }^{1,4}$, ANTONIO TORRES-GARCÍA ${ }^{3,4}$ and PILAR INIESTA ${ }^{1,4}$ \\ ${ }^{1}$ Department of Biochemistry and Molecular Biology, Faculty of Pharmacy, Complutense University; \\ ${ }^{2}$ Center for Biomedical Research Network on Diabetes and Associated Metabolic Diseases; \\ ${ }^{3}$ Digestive Surgery Service, San Carlos Hospital; ${ }^{4}$ Sanitary Research Institute of San Carlos Hospital (IdISSC); \\ ${ }^{5}$ Endocrinology and Nutrition Service, San Carlos Hospital, Madrid 28040, Spain
}

Received November 30, 2020; Accepted April 8, 2021

DOI: $10.3892 / \mathrm{mco} .2021 .2346$

\begin{abstract}
The risk of colorectal cancer (CRC) development has been associated with telomere dysfunction and obesity. However, clinical relevance of these parameters in CRC prognosis is not clear. Therefore, the aim of the present study was to evaluate the impact of obesity and telomere status in the prognosis of patients affected by CRC and submitted to curative surgical treatment. According to published data, this is the first work in which obesity and telomere status are jointly considered in relation to CRC prognosis. A prospective study including 162 patients with CRC submitted to curative surgical treatment was performed. Subjects were classified according to their BMI. Telomere status was established through telomere length and telomerase activity evaluation. Statistical analyses were performed using the SPSS software package version 22. Telomere shortening was inversely associated with BMI in patients with CRC. Notably, among patients with CRC, subjects with obesity exhibited less shortening of tumor telomeres than non-obese patients $(\mathrm{P}=0.047)$. Patients with shorter telomeres, both in the tumor (median telomere length $<6.5 \mathrm{~kb}$ ) and their non-tumor paired tissues (median telomere length $<7.1 \mathrm{~kb}$ ), had the best clinical evolution, regardless of the Dukes' stage of cancers $(\mathrm{P}=0.025$, for tumor samples; $\mathrm{P}=0.003$, for non-tumor samples). Additionally, subjects with a $\mathrm{BMI}>31.85 \mathrm{~kg} / \mathrm{m}^{2}$ showed the worse clinical outcomes
\end{abstract}

Correspondence to: Professor Pilar Iniesta, Department of Biochemistry and Molecular Biology, Faculty of Pharmacy, Complutense University, Ramón y Cajal Square s/n (Ciudad Universitaria), Madrid 28040, Spain

E-mail: insepi@ucm.es

Key words: obesity, telomere, telomerase, colorectal cancer, prognosis compared with subjects with other BMI values. Interestingly, the impact of BMI showed sex dependence, since only the group of men displayed significant differences in CRC prognosis in relation to obesity status $(\mathrm{P}=0.037)$. From the results of the present study, based on a multivariate prediction model to establish prognosis, it was concluded that telomere length is a useful biomarker to predict prognosis in patients with CRC. Regardless of BMI values, the improved clinical evolution was associated with shorter telomeres. The impact of BMI seems to be associated with other factors, such as sex.

\section{Introduction}

Obesity is an emerging and increasingly prevalent condition in the Western world. It is associated with well-known metabolic disorders, leading to the development of different diseases, including cancer (1). Chronic inflammation, a typical feature of obesity, increases the imbalance of the tissue microenvironment, promoting the appearance of the preneoplastic status. In fact, the increase in white adipose tissue (WAT) affects the development of the disease due to the release of several adipokines, interleukins and other cytokines by this type of tissue (2). These factors cause greater hormonal signaling, increased proliferation and survival of adjacent and distal cells which could induce tumorigenesis (3). There is convincing evidence that excess body weight is associated with an increased risk for cancer of at least 13 anatomic sites, including colorectal (4). Thence, the relationship between body mass index (BMI) and risk of colorectal cancer (CRC) has been the focus of several investigations $(4,5)$, and these studies have shown that an increase in BMI is related to a higher incidence of CRC. However, only few of the publications have shown poorer results for patients with obesity $(6,7)$ and, therefore, the relationship between obesity and the prognosis of CRC has not been widely studied.

As is known, one of the hallmarks in the development of tumorigenesis is the gain of replicative immortality through the 
maintenance of telomere length (8). Telomeres are a complex of nucleoproteins, which have a fundamental role in the protection of genomic DNA, and they are shortened with each cell cycle of replication (9). Individuals with short telomeres should be at increased risk for cancer, since short telomeres lead to genomic instability-a hallmark of cancer. However, individuals with long telomeres also display an increased risk for major cancers, thus creating a cancer-telomere length paradox (10). A recent study investigating relative telomere length in white blood cells provides evidence in support of longer telomeres being associated with a higher risk of colorectal cancer, particularly rectal cancer (11). In most cases, shortened telomeres induce a cell-cycle arrest or trigger apoptosis, although for those cells that bypass such signals during tumour progression, a critical length threshold is reached at which telomere dysfunction may ensue (12). Therefore, telomere attrition resulting in replicative senescence, simultaneously by passing cell cycle checkpoints, is a hallmark of malignant transformation of the cell (13).

Telomerase enzyme is the main responsible for telomere maintenance (14). The majority of tumors (80-85\%) sustain their capacity to grow indefinitely through the ectopic expression of telomerase, as shown in previous studies $(15,16)$. Telomerase is almost ubiquitous in advanced solid cancers, including $\mathrm{CRC}$, and its expression is essential for cell immortalization (13).

Many studies have shown that telomere dysfunction, understood as a critical telomere shortening, has a dual role in the development of the tumor, since it can act as a tumor suppressor or an oncogenic factor, depending on the cellular context (17). Attrition of telomeres induces the loss of telomere function, which increases genomic instability and activates regulatory molecules such as p53 that lead to senescence and cell death $(18,19)$. On the other hand, this genomic instability causes mutations which could lead to tumorigenesis (20). In addition, it has been described that shorter telomeres are found in patients with higher BMI values (21), due to the impact of oxidative stress and inflammatory processes, suggesting that obesity is related to shortening of telomeres and potentially promotes colorectal carcinogenesis (22-24).

Previous reports, in order to help clinicians optimize their practice, considered crucial to introduce more effective tools that will improve not only early diagnosis, but also prediction of the most likely progression of the disease and response to chemotherapy. In this context, telomere length and telomerase activity have been included among the promising emerging biomarkers in CRC monitoring (25).

The exposed data highlight the importance of studying how obesity influences telomere function and its potential role as a predictor of prognosis in CRC. We report a multivariate predictor model for CRC prognosis. The main novelty of the present work consists of jointly analyzing obesity and telomere status regarding prognosis of CRC patients submitted to curative intention surgery treatment.

\section{Materials and methods}

Patients and tissue samples. One hundred and sixty-two CRC samples and its paired non-tumor tissue samples, used as controls, were obtained from patients who had undergone potentially curative surgery at San Carlos Hospital in Madrid, Spain, along the last 10 years. Paired samples of non-tumor tissues located at least $10 \mathrm{~cm}$ from the margin of the tumor were obtained and confirmed microscopically.

After surgical resection, all tissue samples were instantly frozen in liquid nitrogen and stored at $-80^{\circ} \mathrm{C}$ until processed. Cryostat-sectioned, hematoxylin and Eosin (H\&E) stained samples from each tumor block were examined microscopically by two independent pathologists to confirm the presence of $\geq 80 \%$ tumor cells. Tumors were pathologically staged according to the modification of the original staging scheme of Dukes by Turnbull et al (26). Location of the tumor, grade of differentiation and other clinical-pathological features were also recorded.

Cases were collected independently from gender, age of the patient or tumor stage, and no patient had received previous chemo- or radiotherapy before diagnosis and inclusion in the study. Of all patients included in this study, $24(14,4 \%)$ had diabetes, $7(4,3 \%)$ had previously been diagnosed with an autoimmune disorder, and 26 (16\%) showed hyperlipidemia.

The mean follow-up period of the series was 5 years (range, 1-147 months). Follow-up was defined by the elapsed time between surgery and either last clinical evaluation of the patient during the study period or death of the patient. Recurrence was defined as the appearance, during follow-up, of any local or distant lesion related to the tumoral process. Disease free survival (DFS) was assessed from the day of surgery until confirmed recurrence.

Written informed consent was obtained from patients prior to investigation. This study was approved by the Ethical Committee of the Hospital (C.I. 15/180-E FIS, 24/04/2015), assuring the patients the confidentiality of their data.

Telomere length and telomerase activity evaluation. The mean telomere length (MTL) values were obtained by the Terminal Restriction Fragment (TRF) length procedure. TRF measurement was performed using Telo TTAGGG Telomere Length Assay kit, cat. no. 12209136001 (Roche Applied Science) as previously described (9). TRF lengths for tumor and control tissues were determined by comparing the signals relative to a standard molecular weight using Image Gauge software version 3.46 (Fujifilm). The TRF length ratio was determined as the ratio of the length of tumor tissue TRF and their paired normal tissue TRF (T/N ratio). Shortening or lengthening of TRFs was defined if the TRF length of tumor tissues was shorter or longer than the corresponding non-tumor tissues, respectively.

In colorectal samples, telomerase was measured using the Telomeric Repeat Amplification Protocol (TRAP)-based telomerase polymerase chain reaction (PCR)-enzyme-linked immunosorbent assay (ELISA) kit, cat. no. 11854666910 (Roche Applied Science), which allowed us to establish a semiquantitative assay (27). Thus, considering that the cut-off for TRAP-ELISA negativity corresponds to an optical density $\mathrm{OD}_{450 \mathrm{~nm}}<0.2$, all samples with $\mathrm{OD}_{450 \mathrm{~nm}} \geq 0.2$ were considered telomerase positive (28).

Statistical analysis. Statistical analyses were performed using the SPSS software package version 22 (SPSS Inc.). Differences between two or more groups of study were calculated using 


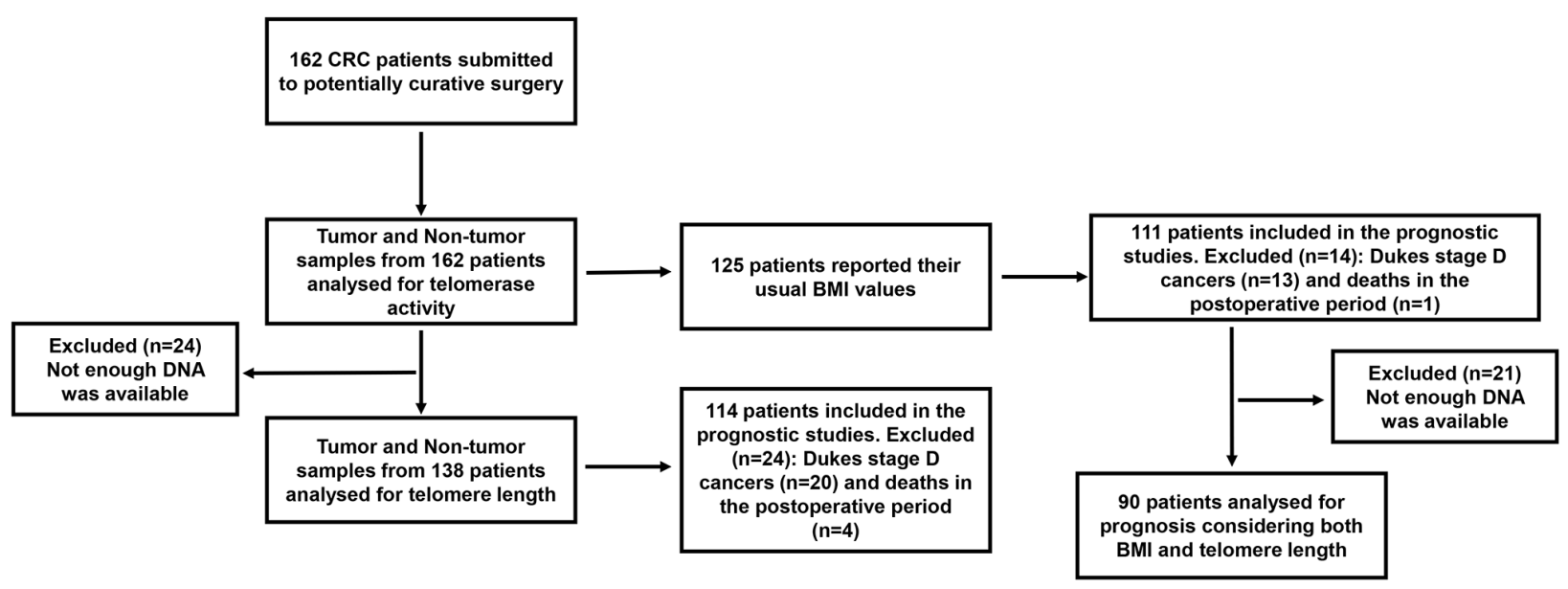

Figure 1. Flow diagram of the process used in the present study. The number of patients involved in each one of the studies, the number of patients excluded, and the causes of exclusion are also specified.

parametric tests, in the case of normality data (ANOVA following by the Bonferroni test for comparing multiple groups) or non-parametric tests, in the case that there were no normality data (Kruskal-Wallis and Mann-Whitney U test). The normality of the data was investigated using the Kolmogorov-Smirnov test and the homoscedasticity conditions of the variables used in this study using the Levene's test for equality of variances. To compare the means of two related variables, Wilcoxon test was executed. Chi-square test was employed to compare categorical, and correlations were assessed by Spearman test; P-values $<0.05$ were considered statistically significant.

Disease-free survival (DFS) was calculated using the Kaplan-Meier method and differences were evaluated by the Log-rank test. Patients with Dukes' D-stage tumors or those who died in the postoperative period were excluded from the survival analysis. The mark of censored data indicated the end of an individual follow-up period. The potential prognostic impact of the variables considered in this work was evaluated jointly by Cox multivariate regression analyses. Cutoff Finder Web Application (http://molpath.charite. de/cutoff/) (29) was used to determine the cut-off points for prognosis analysis.

\section{Results}

Groups of patients. A flow diagram of the progress through the different studies developed in the present work has been included in Fig. 1. Both the number of patients involved in each one of the studies and the number of patients excluded are indicated. Causes of exclusion are also specified.

Of all 162 CRC patients included in this study, 82 were females and 80 males, with a mean age of $70.6 \pm 0.9$ years. Mean age was comparable among both genders (female: $70.3 \pm 1.4$ years vs. male: $71.0 \pm 1.2 ; \mathrm{P}=0.905$ ).

Patients were classified according to their BMI values following the criteria of the World Health Organization (WHO). Thus, patients with BMI $<25 \mathrm{~kg} / \mathrm{m}^{2}$ were considered to have normal weight; patients with BMI $\geq 25 \mathrm{~kg} / \mathrm{m}^{2}$ and $\leq$ $29.9 \mathrm{~kg} / \mathrm{m}^{2}$, as overweight; and the ones with BMI $\geq 30 \mathrm{~kg} / \mathrm{m}^{2}$ were defined as with obesity. BMI data was available for 125 cases, 63 females and 62 males. Overall, 34 patients showed 'normal weight'; 57 were classified as 'overweight'; and 34 were cataloged as 'people with obesity', without significant gender differences $(\mathrm{P}=0.286)$.

Groups of patients established considering BMI values did not show significant associations with Dukes' stage $(\mathrm{P}=0.239)$, nor with tumor location $(\mathrm{P}=0.347)$ (Table I).

Telomere function analysis. Telomere function was investigated through telomere length determination, both in tumor (T) and non-tumor (NT) samples from 138 patients, and by telomerase activity evaluation in paired T and NT samples from 162 subjects. MTL in tumor tissues was $6.11 \pm 0.20 \mathrm{~kb}$, whereas in non-tumor tissues MTL was $8.22 \pm 0.27 \mathrm{~kb}$. A positive correlation was detected between telomere length in tumor and its paired non-tumor samples $(\mathrm{r}=0.501, \mathrm{P}<0.001)$. Moreover, an inverse correlation was found between non-tumor and tumor telomere length and the age of patients $(\mathrm{r}=-0.170, \mathrm{P}=0.028$ for non-tumor tissues, and $\mathrm{r}=-0.155$; $\mathrm{P}=0.041$ for tumor samples).

The mean value for telomere length in tumor and non-tumor samples was correlated with Dukes' stage, as the lowest mean values were observed in the earliest Dukes' stages $(\mathrm{P}=0.032$ for tumor samples) (Table II). Overall, telomere shortening was detected in $\mathrm{CRC}$, as demonstrated by the $\mathrm{T} / \mathrm{N}$ ratio values $(0.78 \pm 0.02)$. $\mathrm{T} / \mathrm{N}$ ratio values were significantly associated with BMI; in fact, patients with obesity and CRC showed less shortening of tumor telomeres $(0.85 \pm 0.05)$ than non-obese patients affected by CRC $(0.72 \pm 0.03)(\mathrm{P}=0.047)$.

Telomerase activity was positive in $121(75.9 \%)$ out of the 162 cases, while 41 (24.1\%) of the CRC were telomerase negative. As shown in Table II, a significantly higher proportion of tumors of the right colon (45.1\%) were negative for telomerase, compared to other locations of cancers (18.9\% of tumors from the left colon, or $14.7 \%$ of tumors from the rectum), with significant differences $(\mathrm{P}<0.001)$.

Survival studies. Regarding telomere status, the outcomes of the patients were analyzed considering the optimal cut-off values for the mean length of the tumor telomeres, as established in a previous publication of our research team (12). 
Table I. BMI groups and clinicopathological variables in patients with colorectal cancer.

\begin{tabular}{|c|c|c|c|c|c|}
\hline Variable & Cases, $n(n=125)$ & $\begin{array}{c}\text { Normoweight } \\
(\mathrm{BMI}<25), \mathrm{n}(\mathrm{n}=34)\end{array}$ & $\begin{array}{c}\text { Overweight } \\
(\mathrm{BMI}=25-29.9), \mathrm{n}(\mathrm{n}=57)\end{array}$ & $\begin{array}{c}\text { Obesity }(\mathrm{BMI} \geq 30), \\
\mathrm{n}(\mathrm{n}=34)\end{array}$ & $\begin{array}{l}\text { P-value } \\
\left(\chi^{2} \text { test }\right)\end{array}$ \\
\hline Sex & & & & & 0.286 \\
\hline Female & 63 & 15 & 27 & 21 & \\
\hline Male & 62 & 19 & 30 & 13 & \\
\hline Dukes' stage & & & & & 0.232 \\
\hline A & 19 & 7 & 6 & 6 & \\
\hline $\mathrm{B}$ & 60 & 13 & 34 & 13 & \\
\hline $\mathrm{C}$ & 33 & 11 & 10 & 12 & \\
\hline $\mathrm{D}$ & 13 & 3 & 7 & 3 & \\
\hline Tumor location & & & & & 0.250 \\
\hline Right colon & 46 & 11 & 22 & 13 & \\
\hline Left colon & 33 & 9 & 19 & 5 & \\
\hline Rectum & 46 & 14 & 16 & 16 & \\
\hline
\end{tabular}

Table II. Telomere status and clinicopathological variables in patients with colorectal cancer.

\begin{tabular}{|c|c|c|c|c|c|c|c|c|c|}
\hline \multirow[b]{2}{*}{ Variable } & \multicolumn{5}{|c|}{ Telomere length (kilobase pairs; mean \pm standard error) } & \multicolumn{4}{|c|}{ Telomerase activity } \\
\hline & $\begin{array}{l}\text { Cases, } n \\
(n=138)\end{array}$ & $\begin{array}{l}\text { Tumor } \\
\text { samples }\end{array}$ & $\begin{array}{l}\text { P-value } \\
\text { (test) }\end{array}$ & $\begin{array}{c}\text { Non-tumor } \\
\text { samples }\end{array}$ & $\begin{array}{l}\text { P-value } \\
\text { (test) }\end{array}$ & $\begin{array}{l}\text { Cases, } n \\
(n=162)\end{array}$ & $\begin{array}{l}\text { Positive, } n \\
\quad(n=121)\end{array}$ & $\begin{array}{l}\text { Negative, } n \\
\quad(n=41)\end{array}$ & $\begin{array}{l}\text { P-value } \\
\left(\chi^{2} \text { test }\right)\end{array}$ \\
\hline Sex & & & 0.423 & & 0.908 & & & & 0.526 \\
\hline Female & 73 & $5.93 \pm 0.29$ & $\begin{array}{l}\text { (Mann- } \\
\text { Whitney } \\
\text { U test) }\end{array}$ & $8.14 \pm 0.36$ & $\begin{array}{l}\text { (Mann- } \\
\text { Whitney } \\
\text { U test) }\end{array}$ & 82 & 63 & 19 & \\
\hline Male & 65 & $6.21 \pm 0.30$ & & $8.43 \pm 0.48$ & & 80 & 58 & 22 & \\
\hline Dukes' stage & & & 0.032 & & 0.198 & & & & 0.784 \\
\hline A & 19 & $4.86 \pm 0.34$ & $\begin{array}{l}\text { (Kruskall- } \\
\text { Wallis test) }\end{array}$ & $7.08 \pm 0.56$ & $\begin{array}{l}\text { (Kruskall- } \\
\text { Wallis test) }\end{array}$ & 22 & 18 & 4 & \\
\hline $\mathrm{B}$ & 62 & $5.89 \pm 0.30$ & & $8.09 \pm 0.39$ & & 66 & 50 & 16 & \\
\hline $\mathrm{C}$ & 37 & $6.71 \pm 0.42$ & & $9.23 \pm 0.74$ & & 44 & 32 & 12 & \\
\hline $\mathrm{D}$ & 20 & $6.55 \pm 0.57$ & & $8.22 \pm 0.68$ & & 30 & 21 & 9 & \\
\hline Tumor location & & & 0.075 & & 0.060 & & & & $<0.001$ \\
\hline Right colon & 43 & $5.40 \pm 0.32$ & $\begin{array}{l}\text { (Kruskall- } \\
\text { Wallis test) }\end{array}$ & $8.36 \pm 0.70$ & $\begin{array}{l}\text { (Kruskall- } \\
\text { Wallis test) }\end{array}$ & 51 & 28 & 23 & \\
\hline Left colon & 33 & $6.60 \pm 0.53$ & & $9.34 \pm 0.58$ & & 37 & 30 & 7 & \\
\hline Rectum & 62 & $6.24 \pm 0.28$ & & $7.65 \pm 0.30$ & & 74 & 63 & 11 & \\
\hline
\end{tabular}

With regard to the CRC population included in the present manuscript, patients whose tumors had a MTL $<6.5 \mathrm{~kb}$ showed the best clinical evolution (Fig. 2A), regardless of Dukes' stage of the tumor, as corroborated by Cox's multivariate analysis (Table III). Therefore, colorectal tumors with an MTL $<6.5 \mathrm{~kb}$ conferred a decreased relative risk of recurrence, more than five times lower than the one of tumors showing an MTL $\geq 6.5 \mathrm{~kb}(\mathrm{RR}=0.169,95 \% \mathrm{CI}=0.036-0.700 ; \mathrm{P}=0.025)$.

Moreover, when survival was analyzed considering the MTL data in non-tumor tissues, a better clinical evolution was found in the group of patients who showed MTL values $<7.1 \mathrm{~kb}(\mathrm{P}=0.003)$ (Fig. 2B). It was not mathematically possible to establish the Cox multivariate study considering MTL data in non-tumor tissues, because one subset of events was empty (no cases of recurrence within the group of MTL $<7.1 \mathrm{~kb}$ ), and we would have obtained an undefined value for RR.

Concerning BMI, the optimal cut-off values for the BMI were calculated using the Cutoff Finder Web Application (24). Patients with a BMI $\geq 31.85$ showed a significantly worse prognosis compared to the group of patients with a BMI $<31.85$ $(\mathrm{P}=0.034)$ (Fig. 3). These results were not independent of the gender of the patients included in this study.

As shown in Fig. 4, males with a BMI greater than or equal to 31.85 showed a significantly worse prognosis compared 
Table III. Multivariate Cox regression analysis in patients with CRC.

\begin{tabular}{lccc}
\hline & \multicolumn{3}{c}{ Multivariate analysis } \\
\cline { 2 - 4 } Variable & P-value & RR & CI \\
\hline Dukes' stage (A + B vs. C) & 0.004 & 4.690 & $1.660-13.240$ \\
Tumor MTL in CRC $(<6.5 \mathrm{~Kb}$ vs. $\geq 6.5 \mathrm{~Kb})$ & 0.025 & 0.169 & $0.036-0.800$ \\
\hline
\end{tabular}

MTL, mean telomere length; RR, relative risk; CI, confidence interval; CRC, colorectal cancer.

A

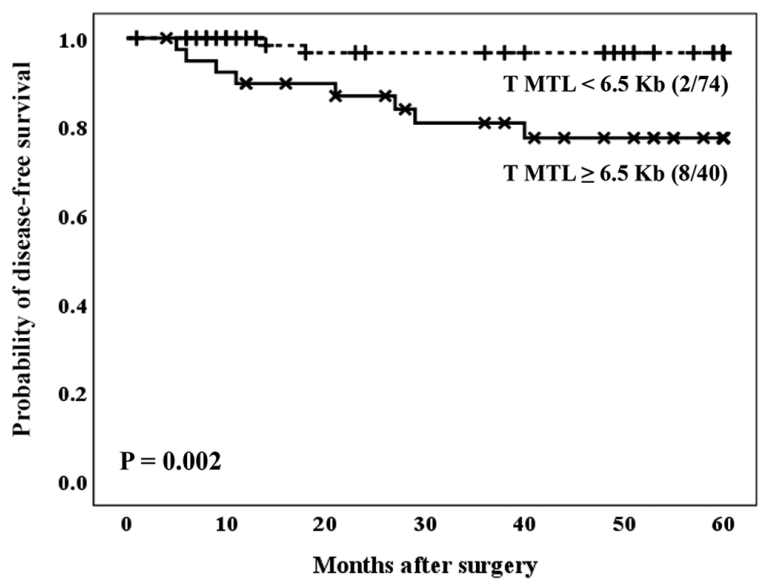

B

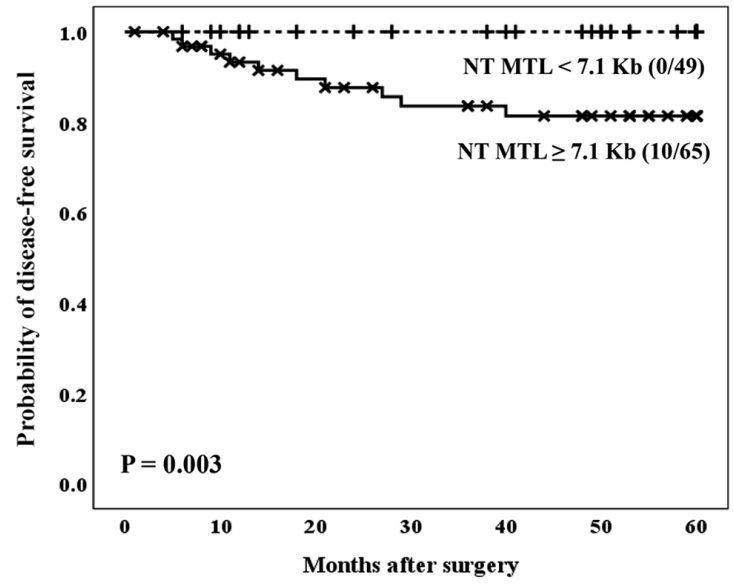

Figure 2. Kaplan-Meier plots of disease-free survival considering telomere length. Kaplan-Meier survival curves in relation to (A) T MTL and (B) NT MTL in the colorectal cancer population ( $\mathrm{n}=114$ patients). Numbers in brackets represent cases with tumor recurrence and crosses indicate censored data. Statistical differences were evaluated using the log-rank test. T MTL, mean telomere length in tumor samples; NT MTL, mean telomere length in non-tumor samples.

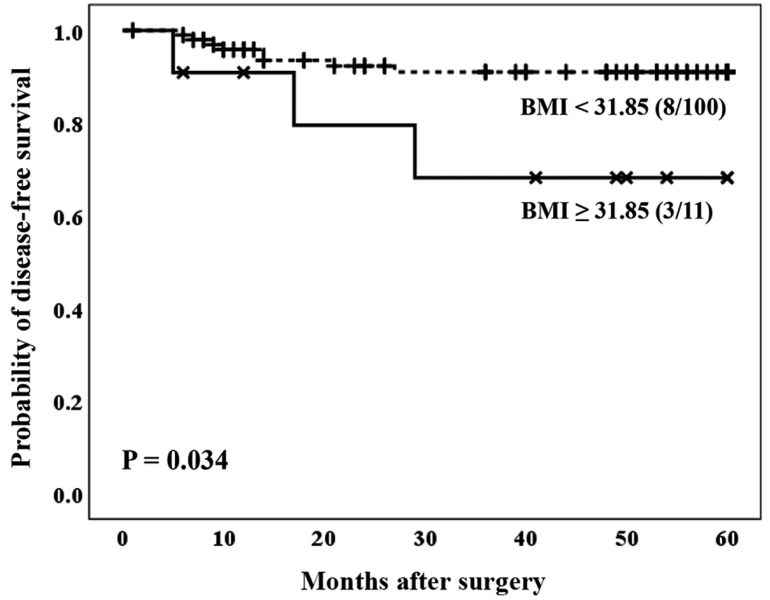

Figure 3. Kaplan-Meier plot of disease-free survival considering BMI. Kaplan-Meier survival curves in relation to a BMI of $31.85 \mathrm{~kg} / \mathrm{m}^{2}$ in patients affected by colorectal cancer ( $\mathrm{n}=111$ subjects). Numbers in brackets represent cases with tumor recurrence and crosses indicate censored data. Statistical differences were evaluated using the log-rank test.

to males with a BMI lower than $31.85(\mathrm{P}=0.037)$ (Fig. 4A). For females, however, such differences were not evident $(\mathrm{P}=0.388)$ (Fig. 4B).

When the two variables (telomere status and BMI) were considered together in relation to the prognosis of the patients affected by the CRC, our results indicated that the telomere status acts as a definitive molecular parameter to establish the prognosis of patients. In fact, independently of the BMI, patients affected by cancer and showing the lowest values of average telomere length, both in tumor and non-tumor samples, showed the best clinical evolution (Fig. 5A and B, for tumor tissues, $\mathrm{P}=0.005$; and Fig. $6 \mathrm{~A}$ and $\mathrm{B}$, for non-tumor tissues, $\mathrm{P}=0.020$ ).

\section{Discussion}

There is growing evidence that inflammation is a central and reversible mechanism through which obesity promotes cancer risk and progression. The tumor promoting effects of obesity occur locally through the inflammation of adipose tissue and the associated alterations in the microenvironment (2). Therefore, the characterization of biomarkers to identify patients with obesity with high-risk CRC seems paramount for an early diagnosis and improvement in the election of the most appropriate therapeutic protocols.

In this context, considering that telomere shortening has been associated with obesity in several studies, and that telomere maintenance is critical for the progression of cancer, our study was carried out considering a large population of CRC patients, with and without obesity, submitted to surgery with curative intention. Results from this work allowed us to demonstrate that telomere status is related to obesity and clinical prognosis. 
A

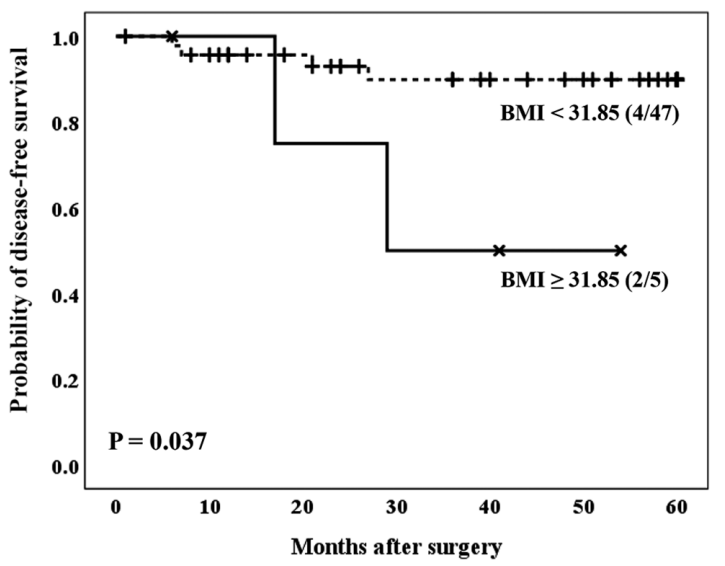

B

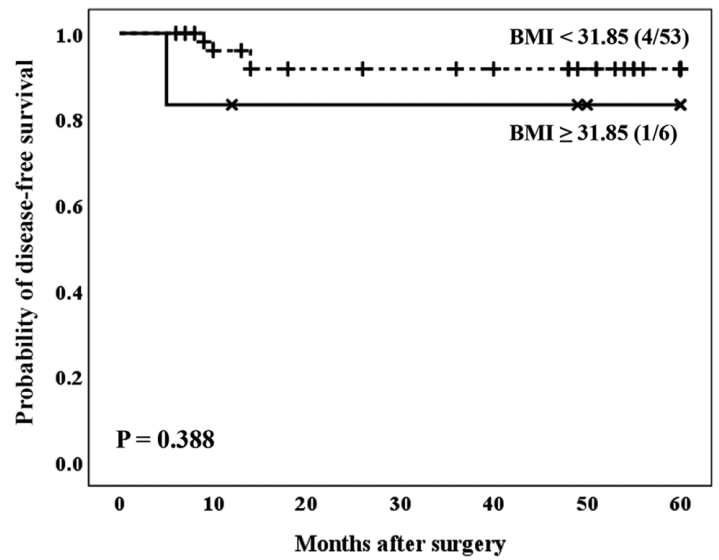

Figure 4. Kaplan-Meier plots of disease-free survival considering BMI and sex of subjects. Kaplan-Meier survival curves in relation to a BMI $<31.85 \mathrm{~kg} / \mathrm{m}^{2}$ or $\geq 31.85 \mathrm{~kg} / \mathrm{m}^{2}$ in (A) male and (B) female patients with colorectal cancer ( $\mathrm{n}=111$ cases). Numbers in brackets represent cases with tumor recurrence and crosses indicate censored data. Statistical differences were evaluated using the log-rank test.

A

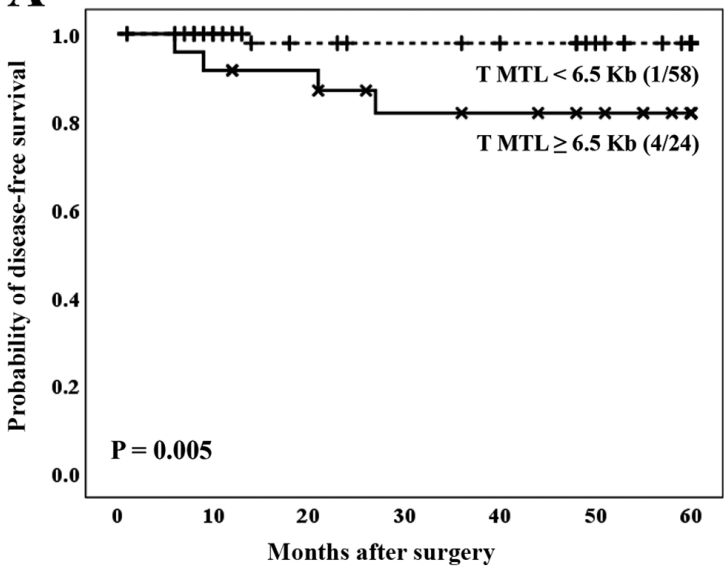

B

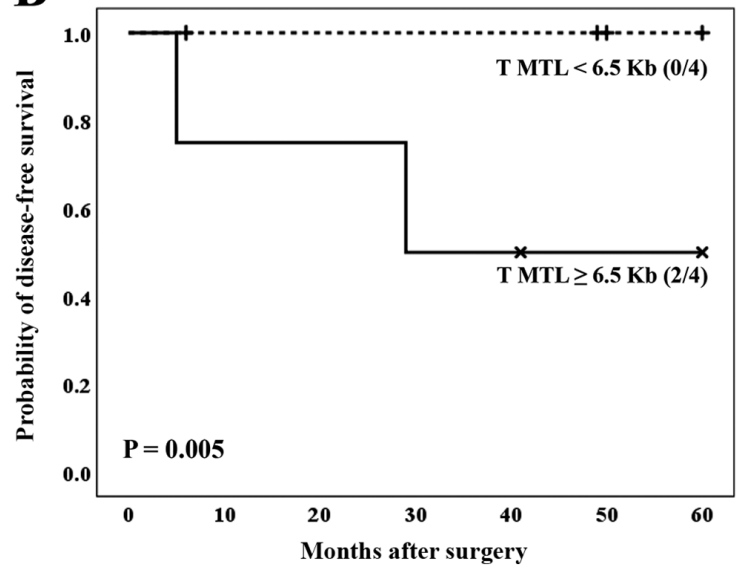

Figure 5. Kaplan-Meier plots of disease-free survival considering T MTL and BMI of patients. Kaplan-Meier curves in relation to T MTL and BMI in the colorectal cancer population ( $\mathrm{n}=90$ patients). (A) BMI $<31.85 \mathrm{~kg} / \mathrm{m}^{2}$ and (B) BMI $\geq 31.85 \mathrm{~kg} / \mathrm{m}^{2}$. Numbers in brackets represent cases with tumor recurrence and crosses indicate censored data. Statistical differences were evaluated using the log-rank test. T MTL, mean telomere length in tumor samples.

Given the technical requirements of the methodologies used in this study, it is necessary to highlight some limitations. On the one hand, not all the usual weight data from patients were available; therefore, there are some cases where the usual BMI could not be calculated. Furthermore, the technique used to evaluate telomere length requires a significant amount of DNA. There were samples in which it was not possible to obtain enough DNA from the available tissues. The number of cases submitted to analysis in each of the determinations included in this work, as well as the reasons for case exclusions, are detailed in the flow diagram in Fig. 1.

Although the activity of telomerase was positive in most of the tumors considered in our study, we detected a significant shortening of telomeres in the tumor samples compared to the mean values of the telomere length observed in the non-tumor paired tissues, as previously reported by others $(16,30)$. Furthermore, according to the results obtained in the present investigation, the lower mean values of telomere length, both in tumors and in their non-tumor paired tissues, were associated with earlier stages of Dukes, in agreement with other groups (31). Thus, these data prove that shorter telomeres are associated with cancers that would, a priori, confer a better prognosis to patients with CRC. In addition, our results indicate that cancers displaying lower values of shortening of telomere length occur in patients with obesity. We consider these data of interest since, although several studies have established a link between obesity and the risk of colon cancer, little is known about the effect of obesity on the outcomes after diagnosis (7).

It has been reported that a BMI greater than $35.0 \mathrm{~kg} / \mathrm{m}^{2}$ at the time of diagnosis in patients with colon cancer is associated with an increased risk of recurrence (7). However, other authors have not confirmed these data, nor a significant correlation between BMI and an increased risk of death in patients with CRC (32). It has also been suggested that BMI prior to diagnosis is an important predictor of survival among patients with non-metastatic CRC (6). More recently, Bhaskaran et al (5) have reported that heterogeneity in the effects of BMI suggests different mechanisms or combinations of mechanisms associated with different tumor locations and in different subgroups of patients. 
A

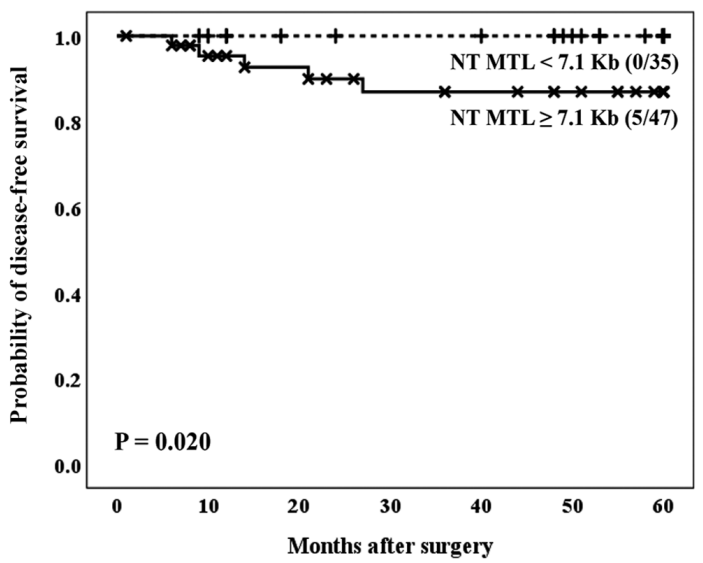

B

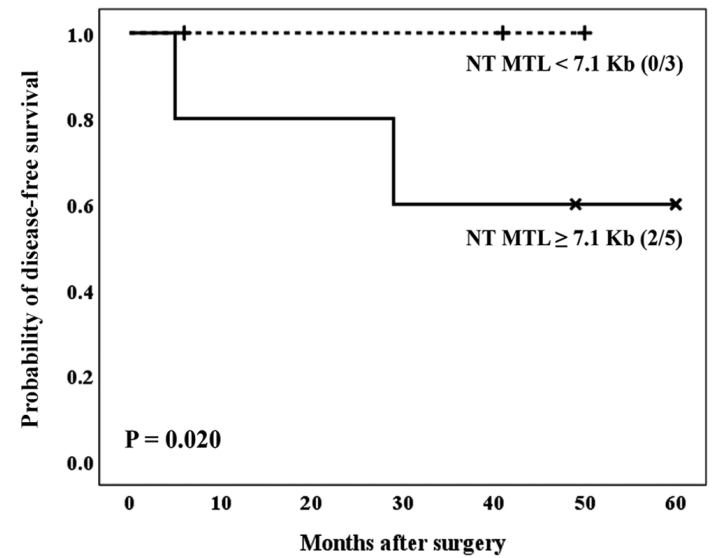

Figure 6. Kaplan-Meier plots of disease-free survival considering NT MTL and BMI of patients. Kaplan-Meier curves in relation to NT MTL and BMI in the colorectal cancer population ( $\mathrm{n}=90$ patients). (A) BMI $<31.85 \mathrm{~kg} / \mathrm{m}^{2}$ and (B) BMI $\geq 31.85 \mathrm{~kg} / \mathrm{m}^{2}$. Numbers in brackets represent cases with tumor recurrence and crosses indicate censored data. Statistical differences were evaluated using the log-rank test. NT MTL, mean telomere length in non-tumor samples.

In our study, the group of patients with BMI values greater than $31.85 \mathrm{~kg} / \mathrm{m}^{2}$, showed a significantly worse clinical prognosis. However, Cox multivariate regression analyses did not demonstrate that these results were independent of the Dukes tumor stage.

Interestingly, our results indicate significant differences according to the gender: When the effect of obesity on the prognosis of patients with CRC was analyzed, these differences were only evident in the male population. Although this fact could be explained by considering the relationship between gender and fat distribution (33), other aspects might be further investigated, including additional genetic, hormonal or molecular mechanisms, in order to explain the effect of obesity on the prognosis of CRC in relation to the gender of patients (34).

As for the telomere status, our data support that the prognosis of patients with CRC, whether with or without obesity, is strongly related to the length of the tumor telomere, being these results independent of the stage of the tumor.

In the present study we have identified a specific length of telomere in non-tumor tissues that seems critical to predict the prognosis of cancer. Therefore, patients with a telomere length less than $7.1 \mathrm{~kb}$ in non-tumor tissues present a better clinical evolution, considering both subjects with and without obesity. At this point, our results would support the idea that carcinogenic cells have a common biological history with normal tissue (35).

These data allow us to hypothesize the possibility that tumor cells with shorter telomeres activate cellular senescence, thereby conferring a more favorable clinical prognosis to patients affected by CRC. In fact, the progressive shortening of telomeres results in the formation of dysfunctional telomeres that compromise tissue proliferation (19).

The shortening of human telomeres has two opposite effects during the development of cancer. On the one hand, the shortening of telomeres can exert a tumor suppressive effect through the arrest of proliferation. On the other hand, the loss of telomere protection can lead to a telomere crisis, which is a state of extensive genomic instability that can promote the progression of cancer (17). Our research team has previously reported data in CRC that support a worse clinical evolution in patients with tumor telomere maintenance (16).

In conclusion, in the present study we have jointly evaluated the prognostic relevance of obesity and telomere status in patients affected by CRC who had undergone surgery of curative intention. Our results demonstrate that the length of telomeres is a useful biomarker to predict the clinical outcome in these subjects. Patients with shorter telomeres, both in the tumor and their non-tumor paired tissues, had the best clinical evolution, independently of the Dukes' stage. Our data allow us to conclude that patients with obesity had a poorer prognosis, however, these results were not independent from the tumor Dukes' stage.

Further investigations are needed to analyze the effect of obesity on the clinical course of CRC in the context of other factors, such as the gender of the patients.

\section{Acknowledgements}

Not applicable.

\section{Funding}

The present study was supported by grants PI15/01199 and PI19/00073 from the Carlos III Institute of Health (Ministerio de Economía y Competitividad), Spain and co-funded by the European Union through the European Regional Development Fund (ERDF) 'A way to make Europe'. Funders did not participate in study design, data collection and analysis, decision to publish, nor preparation of the manuscript.

\section{Availability of data and materials}

The datasets used and/or analyzed during the current study are available from the corresponding author on reasonable request.

\section{Authors' contributions}

ATG and PI conceived and designed the study. SGM, DGG, TFM and ST performed the assays. SDLS, IS, OCV, AB and 
ATG participated in the acquisition and interpretation of data. SGM, DGG, TFM, AB, CDJ, ATG and PI assessed the authenticity of all raw data and analyzed the results. SGM, DGG, SDLS and PI wrote the paper. All authors read and approved the final manuscript.

\section{Ethics approval and consent to participate}

The present study was approved by the Ethical Committee of the San Carlos Hospital (Madrid, Spain). Written informed consent was obtained from patients prior to investigation assuring the confidentiality of their data.

\section{Patient consent for publication}

Not applicable.

\section{Competing interests}

The authors declare that they have no competing interests.

\section{References}

1. Kolb R, Sutterwala FS and Zhang W: Obesity and cancer: Inflammation bridges the two. Curr Opin Pharmacol 29: 77-89, 2016.

2. Iyengar NM, Gucalp A, Dannenberg AJ and Hudis CA: Obesity and cancer mechanisms: Tumor microenvironment and inflammation. J Clin Oncol 34: 4270-4276, 2016.

3. Iyengar NM, Hudis CA and Dannenberg AJ: Obesity and cancer: Local and systemic mechanisms. Annu Rev Med 66: 297-309, 2015

4. Avgerinos KI, Spyrou N, Mantzoros CS and Dalamaga M: Obesity and cancer risk: Emerging biological mechanisms and perspectives. Metabolism 92: 121-135, 2019.

5. Bhaskaran K, Douglas I, Forbes H, dos-Santos-Silva I, Leon DA and Smeeth L: Body-mass index and risk of 22 specific cancers: A population-based cohort study of $5 \cdot 24$ million UK adults. Lancet 384: 755-765, 2014.

6. Campbell PT, Newton CC, Dehal AN, Jacobs EJ, Patel AV and Gapstur SM: Impact of body mass index on survival after colorectal cancer diagnosis: The cancer prevention study-II nutrition cohort. J Clin Oncol 30: 42-52, 2012.

7. Dignam JJ, Polite BN, Yothers G, Raich P, Colangelo L, O'Connell MJ and Wolmark N: Body mass index and outcomes in patients who receive adjuvant chemotherapy for colon cancer J Natl Cancer Inst 98: 1647-1654, 2006.

8. Hanahan D and Weinberg RA: Hallmarks of cancer: The next generation. Cell 144: 646-674, 2011.

9. Wu RA, Upton HE, Vogan JM and Collins K: Telomerase mechanism of telomere synthesis. Annu Rev Biochem 86: 439-460, 2017.

10. Aviv A, Anderson JJ and Shay JW: Mutations, cancer and the telomere length paradox. Trends Cancer 3: 253-258, 2017.

11. Luu HN, Qi M, Wang R, Adams-Haduch J, Miljkovic I, Opresko PL, Jin A, Koh WP and Yuan JM: Association between leukocyte telomere length and colorectal cancer risk in the singapore Chinese health study. Clin Transl Gastroenterol 10: 1-9, 2019.

12. Cleal K, Norris K and Baird D: Telomere length dynamics and the evolution of cancer genome architecture. Int J Mol Sci 19: $482,2018$.

13. Tomasova K, Kroupa M, Forsti A, Vodicka P and Vodickova L: Telomere maintenance in interplay with DNA repair in pathogenesis and treatment of colorectal cancer. Mutagenesis 35: 261-271, 2020.

14. Schmidt JC and Cech TR: Human telomerase: Biogenesis, trafficking, recruitment, and activation. Genes Dev 29: 1095-1105, 2015

15. Garcia-Aranda C, de Juan C, Diaz-Lopez A, Sanchez-Pernaute A, Torres AJ, Diaz-Rubio E, Balibrea JL, Benito M and Iniesta P: Correlations of telomere length, telomerase activity, and telomeric-repeat binding factor 1 expression in colorectal carcinoma. Cancer 106: 541-551, 2006.
16. Fernández-Marcelo T, Sánchez-Pernaute A, Pascua I, De Juan C, Head J, Torres-García AJ and Iniesta P: Clinical relevance of telomere status and telomerase activity in colorectal cancer. PLoS One 11: e0149626, 2016.

17. Maciejowski J and de Lange T: Telomeres in cancer: Tumour suppression and genome instability. Nat Rev Mol Cell Biol 18: 175-186, 2017.

18. Feldser DM and Greider CW: Short telomeres limit tumor progression in vivo by inducing senescence. Cancer Cell 11: 461-469, 2007.

19. Wang Y, Wang X, Flores ER, Yu J and Chang S: Dysfunctional telomeres induce p53-dependent and independent apoptosis to compromise cellular proliferation and inhibit tumor formation. Aging Cell 15: 646-660, 2016.

20. Roger L, Jones RE, Heppel NH, Williams GT, Sampson JR and Baird DM: Extensive telomere erosion in the initiation of colorectal adenomas and its association with chromosomal instability. J Natl Cancer Inst 105: 1202-1211, 2013.

21. Mundstock E, Sarria EE, Zatti H, Mattos Louzada F, Kich Grun L, Herbert Jones M, Guma FT, Mazzola In Memoriam J, Epifanio M, Stein RT, et al: Effect of obesity on telomere length: Systematic review and meta-analysis. Obesity (Silver Spring) 23: 2165-2174, 2015.

22. An R and Yan H: Body weight status and telomere length in U.S. middle-aged and older adults. Obes Res Clin Pract 11: 51-62, 2017.

23. Correia-Melo C, Hewitt G and Passos JF: Telomeres, oxidative stress and inflammatory factors: Partners in cellular senescence? Longev Healthspan 3: 1, 2014.

24. Masi S, Salpea KD, Li K, Parkar M, Nibali L, Donos N, Patel K, Taddei S, Deanfield JE, D'Aiuto F and Humphries SE: Oxidative stress, chronic inflammation, and telomere length in patients with periodontitis. Free Radic Biol Med 50: 730-735, 2011.

25. Nikolouzakis TK, Vassilopoulou L, Fragkiadaki P, Sapsakos T, Papadakis GZ, Spandidos DA, Tsatsakis AM and Tsiaoussis J: Improving diagnosis, prognosis and prediction by using biomarkers in CRC patients. Oncol Rep 42: 2228-2244, 2018.

26. Turnbull RB Jr, Kyle K, Watson FR and Spratt J: Cancer of the colon: The influence of the no-touch isolation technic on survival rates. Ann Surg 166: 420-427, 1967.

27. González-Quevedo R, Iniesta P, Morán A, de Juan C, Sánchez-Pernaute A, Fernández C, Torres A, Díaz-Rubio E, Balibrea JL and Benito M: Cooperative role of telomerase activity and p16 expression in the prognosis of non-small-cell lung cancer. J Clin Oncol 20: 254-262, 2002.

28. Frías C, García-Aranda C, De Juan C, Morán A, Ortega P, Gómez A, Hernando F, López-Asenjo JA, Torres AJ, Benito M and Iniesta P: Telomere shortening is associated with poor prognosis and telomerase activity correlates with DNA repair impairment in non-small cell lung cancer. Lung Cancer 60: 416-425, 2008

29. Budczies J, Klauschen F, Sinn BV, Győrffy B, Schmitt WD, Darb-Esfahani S and Denkert C: Cutoff finder: A comprehensive and straightforward Web application enabling rapid biomarker cutoff optimization. PLoS One 7: e51862, 2012.

30. Bertorelle R, Rampazzo E, Pucciarelli S, Nitti D and De Rossi A: Telomeres, telomerase and colorectal cancer. World J Gastroenterol 20: 1940-1950, 2014

31. Jia $\mathrm{H}$ and Wang $\mathrm{Z}$ : Telomere length as a prognostic factor for overall survival in colorectal cancer patients. Cell Physiol Biochem 38: 122-128, 2016.

32. Meyerhardt JA, Niedzwiecki D, Hollis D, Saltz LB, Mayer RJ, Nelson H, Whittom R, Hantel A, Thomas J and Fuchs CS; Cancer and Leukemia Group B 89803: Impact of body mass index and weight change after treatment on cancer recurrence and survival in patients with stage III colon cancer: Findings from cancer and leukemia group B 89803. J Clin Oncol 26: 4109-4115, 2008.

33. Doleman B, Mills KT, Lim S, Zelhart MD and Gagliardi G: Body mass index and colorectal cancer prognosis: A systematic review and meta-analysis. Tech Coloproctol 20: 517-535, 2016.

34. Cheung WY, Shi Q, O'Connell M, Cassidy J, Blanke CD, Kerr DJ, Meyers J, Van Cutsem E, Alberts SR, Yothers G, et al: The predictive and prognostic value of sex in early-stage colon cancer: A pooled analysis of 33,345 patients from the ACCENT database. Clin Colorectal Cancer 12: 179-187, 2013.

35. Nakamura K, Furugori E, Esaki Y, Arai T, Sawabe M, Okayasu I, Fujiwara M, Kammori M, Mafune K, Kato M, et al: Correlation of telomere lengths in normal and cancers tissue in the large bowel. Cancer Lett 158: 179-184, 2000. 